\title{
Comparison of Lower Limb Muscle Activities by Various Angles of a Medio-lateral Ramp During Gait Sang-Yeol Lee, PT, $\mathrm{PhD}^{\dagger}$ • Soo-Hong Ahn, $\mathrm{PT}^{1}$ \\ Dept. of Physical Therapy, College of Science, Kyungsung University \\ ${ }^{1}$ Dept. of Biomedical Health Science, Dongeui University
}

Received: July 19, 2017 / Revised: July 19, 2017 / Accepted: July 24, 2017

(C) 2017 J Korean Soc Phys Med

\begin{abstract}
| Abstract |
PURPOSE: This study investigated the activities of lower limb muscles according to the angle of a medio-lateral ramp while walking to promote awareness of the risks associated with a medio-lateral ramp.
\end{abstract}

METHODS: This study was conducted on 20 healthy male adults. The muscle activities of the vastus medialis oblique (VMO), vastus lateralis oblique (VLO), tibialis anterior (TA) and peroneus longus (PL) were measured while the subjects were walking on a $3 \mathrm{~m}$ medio-lateral ramp. Five angles (flat, $2^{\circ}, 5^{\circ}, 10^{\circ}$, and $15^{\circ}$ ) were selected for the angle conditions of the experiment on a medio-lateral ramp. The activities were measured during the stance phase only in the middle cycle of a three-cycle walking experiment. The mean value obtained from the three walking tests was used for the analysis.

RESULTS: Results showed that walking on a mediolateral ramp required more muscle activities than walking on a flat surface, through which balanced walking was achieved.

CONCLUSION: Walking on a medio-lateral ramp requires proper muscle activation and control, without which the risks

† Corresponding Author : sjslh486@ks.ac.kr

This is an Open Access article distributed under the terms of the Creative Commons Attribution Non-Commercial License (http://creativecommons.org/licenses/by-nc/3.0) which permits unrestricted non-commercial use, distribution, and reproduction in any medium, provided the original work is properly cited. of injury to the joints of the lower limbs and falls are likely to increase. Therefore, special attention should be given to older people and the disabled under the condition of traversing a ramp.

Key Words: Balance, Lower limb muscle activities, Medio-lateral ramp

\section{Introduction}

Human walking is a repetitive movement resulting from a series of coordinate and delicate movements (Perry, 1992). Falling occurs when any one of these delicate movements is not in harmony or fails to function. The factors that cause falls include internal factors, which occur because of the inability of the body to function, and external factors, which result from external environmental changes (Nevitt et al., 1997). Internal factors are mostly related to injuries and aging, whereas external factors are related to the external environment that causes instability. Ramps and staircases that enhance the convenience of living by tilting the ground are typical examples of instability caused by changes in the external environment (Han et al., 2009).

Ramps are categorized into uphill-downhill ramps, which have the same direction for moving and tilting, and 
medio-lateral ramps, which have different directions for moving and tilting. Given the same direction for moving and tilting in uphill-downhill ramps, the gait pattern of the left and right feet is identical, whereas the gait pattern of the left and right feet is different in medio-lateral ramps (Lee et al., 2017). Therefore, failure in the proper adjustment of the left and right feet can lead to risk of falls. A previous study reported that standing on a medio-lateral ramp at an angle of $10^{\circ}$ caused an imbalance in the multifidus muscles of both legs (Lee, 2017). Moreover, when walking on a medio-lateral ramp, more activities of the gluteus medius were needed for the stance phase performed on a medical ramp as the angle of the ramp increased, and more activities of the peroneus longus muscle were required for the stance phase performed on a lateral ramp (Lee et al., 2017; Kim and Lee, 2017). As shown in previous studies, medio-lateral ramps may increase the risks of ankle sprain and fall.

However, these previous studies failed to measure and compare the medial and lateral slopes during walking and did not compare the overall muscular activities observed in the lower limbs during walking on a flat surface and on a medio-lateral ramp, as the evaluation was performed only on a single muscle type. Therefore, this study aims to measure and compare the activities of various lower limb muscles during walking on a $3 \mathrm{~m}$ medio-lateral ramp.

\section{Methods}

\section{Participants}

This study was conducted on 20 healthy male adults whose predominant side is the right side. The mean age of the subjects was $23.15 \pm 2.14$ years. The mean body weight was $68.65 \pm 3.42 \mathrm{~kg}$, the mean height was $173.75 \pm$ $2.80 \mathrm{~cm}$, and the mean body mass index (BMI) was $22.74 \pm$ 1.07. All subjects voluntarily agreed to participate in the study after learning the details of the study.

\section{Data acquisition and processing}

In this study, the activities of lower limb muscles were measured according to the various angles of a medio-lateral ramp during a single support time while walking. The single support time of each foot corresponding to the medial ramp and the lateral ramp was measured. The section of the single support time was defined as the time from the touchdown of the heel to the lift-off of the toe. To measure the single support time accurately from each foot, a 3D motion analyzer (Motion Analysis, Motion Analysis, USA), which was linked to an electromyogram (EMG) instrument, was used, and the section covering the heel's lowest point up to the point where the big toe lifts from the floor was analyzed. The single support time was measured accurately using the Falcon System (Motion Analysis, USA) consisting of six cameras (Hawk Digital RealTime System, Motion Analysis Inc., USA), and the measurement data were extracted using the Cortex program. The vastus medialis oblique (VMO), vastus lateralis oblique (VLO), tibialis anterior (TA), and peroneus longus (PL) muscles of the extracted segments were measured. For the measurement of muscle activities during gait, the middle gait cycle of the three gait cycles was analyzed. The mean value obtained from three gait tests was calculated to be used in the analysis. The left foot was used for all measurements.

Muscle activities were measured using a surface EMG system (Myosystem TM DTS, Noraxon Inc., USA), and the results of the measurements were analyzed using an EMG analysis program (Biomechanical analysis software MR 3.8, Noraxon Inc., USA). $\mathrm{Ag} / \mathrm{AgCl}$ electrodes (IWC-DTS, 9113A-DTS, Noraxon Inc., USA) were used as surface electrodes, which were attached at intervals of $1 \sim 2 \mathrm{~cm}$. The sampling rate of the EMG signal was set to $1,024 \mathrm{~Hz}$, and filtering was conducted by using a $20 \sim 500$ $\mathrm{Hz}$ band-pass filter and a $60 \mathrm{~Hz}$ notch filter. The collected signals were quantified as root mean square (RMS) values. The maximum voluntary isometric contraction (MVIC) of each muscle to be subjected to the data analysis was 
measured before the experiment, and the data were converted to the percentage MVIC for analysis.

A self-made aluminum ramp, which was manufactured to effectively conduct three cycles of the walking experiment, was used as the experimental ramp in this study.

This study was conducted after obtaining the approval of the Institutional Review Board of Kyungsung University (KSU-17-04-004).

\section{Statistical analysis}

The data obtained in this study were statistically processed using IBM SPSS 23.0. One-way ANOVA was employed to compare the muscle activities during walking on the medio-lateral ramp at five different angles. To determine the differences in the activities according to each slope condition, least significant difference (LSD) was used for the post-hoc test. The statistical significance level a was set to .05 .

\section{Results}

\section{Muscle activities on the medial ramp}

During the stance phase while walking on the medial ramp, the muscle activities of the VMO, TA, and PL were significantly different at each angle $(\mathrm{p}<.05)$ (Table 1$)$. The results of the post-hoc test showed that the muscle activities of the $\mathrm{VMO}, \mathrm{PL}$, and $\mathrm{TA}$ on the $10^{\circ}$ and $15^{\circ}$ ramps were significantly higher than those on the flat surface and the $2^{\circ}$ and $5^{\circ}$ ramps $(\mathrm{p}<.05)$ (Table 1$)$.

\section{Muscle activities on the lateral ramp}

During the stance phase while walking on the lateral ramp, the muscle activities of the VMO, VLO, TA, and PL were significantly different at each angle $(p<.05)$ (Table 2). The results of the post-hoc test showed that the muscle activities of the VMO were significantly higher on the ramps at all angles above $2^{\circ}$ than on the flat surface. The

Table 1. Lower limb muscle activities during the stance phase on the medial ramp

\begin{tabular}{cccccccc}
\hline & Flat & $2^{\circ}$ & $5^{\circ}$ & $10^{\circ}$ & $15^{\circ}$ & $\mathrm{F}$ & $\mathrm{P}$ \\
\hline VMO & $11.9 \pm 3.0$ & $10.6 \pm 2.4$ & $13.8 \pm 1.9$ & $18.2 \pm 4.9^{\dagger}$ & $14.6 \pm 1.5^{\dagger}$ & 16.92 & $.00^{*}$ \\
VLO & $16.7 \pm 5.8$ & $14.8 \pm 4.3$ & $16.5 \pm 6.7$ & $16.8 \pm 5.6$ & $16.8 \pm 3.6$ & .625 & .64 \\
TA & $15.6 \pm 4.6$ & $15.9 \pm 4.5$ & $18.6 \pm 4.6$ & $21.8 \pm 8.1^{\dagger}$ & $22.7 \pm 8.3^{\dagger}$ & 5.34 & $.00^{*}$ \\
PL & $13.7 \pm 8.5$ & $12.4 \pm 4.9$ & $16.1 \pm 7.4$ & $23.7 \pm 7.5^{\dagger}$ & $20.9 \pm 7.2^{\dagger}$ & 8.63 & $.00^{*}$ \\
\hline
\end{tabular}

Mean \pm SD

* : statistically significant, $\mathrm{p}<.05$

NOTE. VMO: Vastus medialis oblique, VLO: Vastus lateralis oblique, TA: Tibialis anterior muscle, PL: Peroneus longus muscle Each value represents the mean (SD). The values with different superscripts $\left(^{+}\right)$in the same column are significantly different $(\mathrm{p}<.05)$ according to LSD.

Table 2. Lower limb muscle activities during the stance phase on the lateral ramp

\begin{tabular}{ccccccccc}
\hline & Flat & $2^{\circ}$ & $5^{\circ}$ & $10^{\circ}$ & $15^{\circ}$ & $\mathrm{F}$ & $\mathrm{p}$ \\
\hline VMO & $11.9 \pm 3.0$ & $15.8 \pm 2.4+$ & $14.8 \pm 2.7+$ & $17.4 \pm 5.6^{\dagger}$ & $15.9 \pm 3.9^{\dagger}$ & 5.86 & $.00^{*}$ \\
VLO & $16.7 \pm 5.8$ & $14.3 \pm 4.3$ & $13.9 \pm 4.9$ & $31.2 \pm 8.0^{\dagger}$ & $21.6 \pm 6.4^{\dagger}$ & 26.81 & $.00^{*}$ & $.00^{*}$ \\
TA & $15.6 \pm 4.6$ & $15.7 \pm 3.4$ & $16.3 \pm 3.8$ & $16.7 \pm 3.6$ & $12.4 \pm 4.4^{\dagger}$ & 3.58 & $.00^{*}$ \\
PL & $13.7 \pm 8.5$ & $14.8 \pm 3.1$ & $15.5 \pm 4.6$ & $17.3 \pm 2.9$ & $74.9 \pm 28.4^{\dagger}$ & 77.28 &
\end{tabular}

Mean \pm SD

* : statistically significant, $\mathrm{p}<.05$

NOTE. Each value represents the mean (SD). The values with different superscripts $\left({ }^{\dagger}\right)$ in the same column are significantly different $(\mathrm{p}<.05)$ according to LSD. 


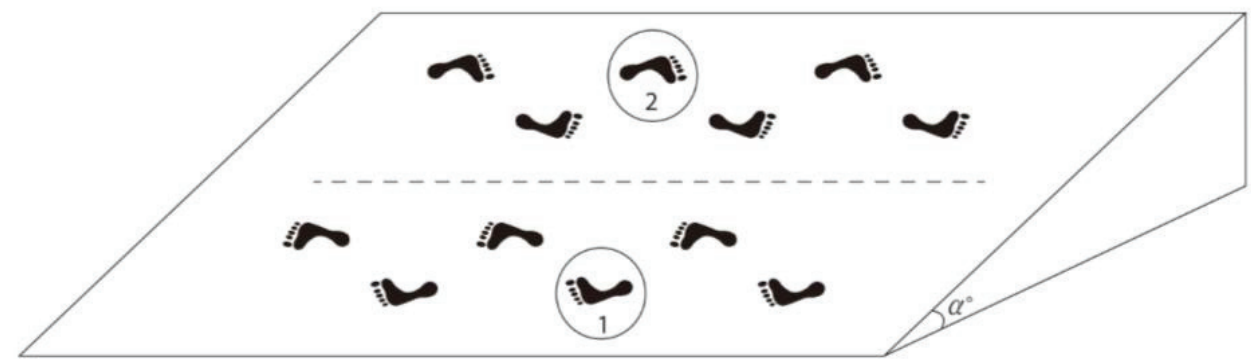

Fig. 1. Experimental conditions of this study. 1) lateral ramp, 2) medial ramp

VLO showed higher activities on the $10^{\circ}$ and $15^{\circ}$ ramps than on the flat surface and on the $2^{\circ}$ and $5^{\circ}$ ramps. TA and PL muscles showed higher activities on the $15^{\circ}$ ramp than on the $2^{\circ}, 5^{\circ}$, and $10^{\circ}$ ramps $(\mathrm{p}<.05)$ (Table 2).

\section{Discussion}

The ramp is an essential environmental condition for human life and is an environmental condition used by all people, including older people and the disabled. Given that the purpose of ramps is to connect the ground with different altitudes without using stairs, researchers are continuously studying uphill and downhill ramps to promote awareness of their risks. Recently, research has been conducted on the environmental risks of the translateral use of ramps (Lee et al., 2017; Lee, 2017; Kim and Lee, 2017).

This study was conducted to analyze the environmental risks of traversing ramps based on the activities of lower limbs. The results indicated that the activities of VMO, VLO, and PL in the lower limb supporting the medial ramp increased as the angle of the ramp increased. These results suggested that the eversion tolerance range of the ankle joint is limited by the shape of the surrounding ligaments and joints (Neumann, 2002). In the environment of the medial-lateral ramp, where the inversion and eversion directions created on the ground are different from those on the walking direction, the gait stability should be achieved through the regulation of diverse muscles. The results also showed that the activities of the lower limb muscles increased at a less steep angle than the angle reported from a previous study (Kim and Lee, 2017). In this previous study, the muscle activities of the gluteus medius increased on a $15^{\circ}$ medial ramp, thus suggesting that the balance control in the lower limb muscles precedes the muscle activation of the gluteus medius. Significant increases in the activities of all three muscle types on the $10^{\circ}$ medial-lateral ramp indicated that, given that an eversion of approximately $10^{\circ}$ is allowed in the transverse tarsal joint (Neumann, 2002), the regulation of more muscles is required when eversion is created on a ramp at an angle higher than $10^{\circ}$.

Moreover, the activities of VMO, VLO, TA, and PL in the lower limb supporting the lateral ramp increased as the angle of the ramp increased. The activities of VMO showed a significant increase even on the $2^{\circ}$ ramp compared with the flat surface. Conversely, the activities of VLO did not show a significant difference until the angle of the ramp reached $10^{\circ}$. The reason why the activities of VMO changed on the lateral ramp at a smaller angle than those of VLO is considered to ensure stability by reducing the bowstringing force on the patellar (Neumann, 2002), which has a greater impact on the knee joint in unstable postures. That is, the knee joint can become unstable on a lateral ramp at an angle as small as $2^{\circ}$. In addition, the activities of PL drastically increased on the $15^{\circ}$ lateral ramp. 
These results suggested that although the transverse tarsal joint allowed an inversion of about $20^{\circ}$, the surrounding ligaments and joints did not form the appropriate shape that could achieve stability, which was different from the case of eversion. Furthermore, the foot performing the stance phase on the lateral ramp during walking was always located below the horizontal plane, thus causing its distance to the ground to be longer than the foot on the upper medial ramp. As a result, the level of acceleration increased, and a higher repulsive force was transmitted from the ground. With the increase in the angle of the ramp, the difference in the height increased. Therefore, the activation of far more muscles is required to stabilize the foot performing the stance phase on the lateral ramp under a specific condition, such as a steeper ramp with different heights (Lee et al., 2017).

Therefore, the results of this study indicated that more muscle activities are required for walking on the mediolateral ramp than on a flat surface. Accordingly, the risk of falls and injuries of the knee joint and ankle joint are likely to increase in older people, who lack such muscle activities (Horak and Nashner, 1986; Mackey and Robinvitch, 2005).

Given that this study was conducted on healthy adult males, generalizing the conclusion of this study to subjects with balance impairment, such as older people and the disabled, is difficult. Therefore, further studies on subjects with balance impairment are needed in the future. In addition, examining the overall mechanisms on the balance control of the body on a medio-lateral ramp is necessary to apply the information obtained from these studies to the establishment of the standards for ramp and crosswalk installations in sloped roads.

\section{Conclusion}

This study was conducted to investigate the effect of medio-lateral ramp angles on lower limb muscles during walking and to bring awareness about the associated dangers of using the ramp. The results showed that walking on both medial and lateral ramps resulted in changes in the lower limb muscles even at a small angle. Therefore, care should be taken while walking on a medio-lateral ramp. In the future, special attention should be paid to older people and the disabled under the condition of traversing a medio-lateral ramp.

\section{Acknowledgements}

This work was supported by the National Research Foundation of Korea (NRF) grant funded by the Korea government (MSIP; Ministry of Science, ICT \& Future Planning) (No. 2017R1C1B5015093).

\section{References}

Han JT, Gong WT, Lee YS. Comparison of muscle activity with lower extremity during stairs and ramp climbing of old adults by EMG. J Kor Soc Phys Ther. 2009;21(1):35-40.

Horak FB, Nashner LM. Central programming of postural movements: adaptation to altered support-surface configurations. J Neurophysiol, 1986;55(6): 1369-81

Kim SC, Lee SY. Gluteus medius muscle activities according to various angle of mediolateral ramp during cross walking and one-leg standing. J Korean Soc Phys Med. 2017;12(2):53-7.

Lee SY, Lee SM, Jung JM. Peroneus longus activity according to various angles of a ramp during cross-ramp walking and one-legged standing. J Back Musculoskelet Rehabil. 2017; Epub ahead of print.

Lee SY. The effects of ratio in the multifidus muscle thickness according to various angle of mediolateral ramp during 
double stance. J Phys Ther Sci. 2017; Pub ahead of print.

Mackey DC, Robinovitch SN. Postural steadiness during quiet stance does not associate with ability to recover balance in older women. Clin Biomech. 2005;20(8):776-83. Neumann DA. Kinesiology of the Musculoskeletal System:
Foundations for Physical Rehabilitation ( $2^{\text {nd }}$ ed). St Louis. Mosby. 2002.

Nevitt MC. Risk factors for recurrent nonsyncopal falls in order persons. Am J Public Health. 1997;87:1677-9.

Perry J. Gait Analysis: Normal and Pathological Function. Thorofare, NJ : SLACK Incorporated. 1992. 